\title{
Sex Disparity in the Effect of Obesity in Hospitalized COVID-19 Patients: A Retrospective Cohort Study From the New York City Metropolitan Area
}

Ashutossh Naaraayan ${ }^{1}$, Abhishek Nimkar ${ }^{1}$, Sushil Pant ${ }^{1}$, Amrah Hasan ${ }^{1}$, Momcilo Durdevic ${ }^{1}$, Henrik Elenius ${ }^{1}$, Corina Nava Suarez ${ }^{1}$, Stephen Jesmajian ${ }^{1}$

1. Internal Medicine, Montefiore New Rochelle Hospital, Albert Einstein College of Medicine, New Rochelle, USA

Corresponding author: Ashutossh Naaraayan, ashu.newroc@gmail.com

\begin{abstract}
Introduction: Obesity has been recognized as a risk factor for poor outcomes in coronavirus disease 2019 (COVID-19) illness. We analyzed the impact of patient characteristics including obesity on hospital mortality and specifically analyzed the effect of obesity by body mass index (BMI) class and by sex.

Methods: This retrospective case series included adult patients consecutively hospitalized with confirmed COVID-19 illness between March 12, 2020 and May 13, 2020, at a teaching hospital in the New York City (NYC) metropolitan area. Data were manually extracted from electronic health records by the authors and included demographics, comorbidities, laboratory parameters, and outcomes (hospital mortality or discharge). We used univariable and multivariable logistic regression methods to explore the risk factors associated with in-hospital death.
\end{abstract}

Results: Some 348 patients were included in this study, of whom 207 were discharged and 141 died in the hospital. Multivariable regression showed increasing odds of in-hospital death with older age and excess weight. Interestingly obesity increased mortality in women [odds ratio (OR) 4.4, confidence interval (CI) (1.4-13.5) $\mathrm{p}=0.01]$ but not among men [OR 1.4, CI (0.5-3.6) $\mathrm{p}=0.5$ ]. Among women, the effect of excess weight on mortality was seen in a "dose-effect" fashion, with increasingly higher odds of mortality from progressively worsening obesity (OR ranging between 2.7 and 6.9). Out of all the comorbidities, only obesity positively correlated with peak levels of C-reactive protein (CRP).

Conclusion: Advancing age is a risk factor for in-hospital death during COVID-19 illness. Obese women could be at a higher risk for mortality due to COVID-19 and should take extra precautions to prevent contamination by social distancing and other measures. Immunomodulators may be more effective in obese women affected by COVID-19. Further studies are needed to help elucidate this association.

Review began 04/28/2021 Review ended 05/25/2021 Published 05/25/2021

\section{() Copyright 2021}

Naaraayan et al. This is an open access article distributed under the terms of the Creative Commons Attribution License CC-BY 4.0., which permits unrestricted use, distribution, and reproduction in any medium, provided the original author and source are credited.
Categories: Internal Medicine, Infectious Disease, Public Health

Keywords: covid-19, sex differences, disease mortality, body mass index: bmi, age and ageing

\section{Introduction}

At the time of writing, 141 million cases of coronavirus disease 2019 (COVID-19) caused by severe acute respiratory syndrome coronavirus 2 (SARS-CoV-2) have been described worldwide, resulting in three million deaths [1]. Initially reported as a local outbreak in Wuhan, China on December 31, 2019, this illness caused by a novel coronavirus rapidly transitioned to become a global threat and was declared a pandemic by the World Health Organization on March 11, 2020. To date, laboratory-confirmed cases have been documented from 192 countries across six continents. Through March and April 2020, the New York City (NYC) metropolitan region was the epicenter for COVID-19 [2]. A concerning number of cases and deaths are now being reported globally from India, Brazil, and other nations.

Although COVID-19 illness mainly manifests as fever, cough, myalgia and fatigue, illness severity can run the spectrum from asymptomatic or paucisymptomatic infection ( $80 \%$ of patients) to severe infection requiring hospitalization, at times with life-threatening sepsis, multi-organ failure, and death [3]. It is crucial to identify the demographic and comorbid patient factors that contribute to severe illness causing hospitalization and death. Here, we present data analyzing patient risks for mortality during COVID-19 hospitalizations, from one of the earliest epicenters in the United States [2]. Obesity has emerged as a major risk factor for mortality in COVID-19 [4]. We specifically investigated the impact of different obesity classes on mortality in hospitalized patients and the impact of obesity in patients with COVID-19 by sex.

\section{Materials And Methods}




\section{Study design and participants}

This retrospective case series included adult (>18 years old) patients consecutively hospitalized with confirmed COVID-19 illness between March 12, 2020 and May 13, 2020, at a 242-bed teaching community hospital in the NYC metropolitan area. The hospital serves approximately 250,000 people in southern Westchester County, New York. Cases were confirmed through positive results for the SARS-CoV-2 virus by reverse-transcriptase-polymerase-chain-reaction testing of a nasopharyngeal swab specimen.

Outcome data were followed up until May 22, 2020. Of the 365 patients admitted during the study period, those who were transferred to another facility for tertiary level care $(n=5)$, those who left against medical advice $(\mathrm{n}=2)$ were excluded from the analysis. We did not have a good representative sample of underweight patients $(n=10)$ and thus excluded these patients from analysis. After the above-mentioned exclusions, the final analysis included 348 patients.

\section{Data collection}

Data were manually extracted from electronic health records by the authors and included demographics, comorbid conditions, certain laboratory parameters, and outcomes (hospital mortality or discharge). Laboratory data for the highest values of peripheral blood C-reactive protein (CRP) (peak-CRP) and D-dimer (peak-D-dimer) levels during hospitalization were recorded. Three authors (AN, AN, and SP) independently reviewed the data for accuracy.

\section{Definition of patient characteristics}

Comorbidities derived from the patients or nursing home $(\mathrm{NH})$ transfer forms were extracted from physician documentation on the electronic health records. The race was classified into one of the five categories: Caucasian, African-American, Hispanic, Asian or Pacific-Islander, and others. Cardiac disease was defined as chronic heart conditions including coronary artery disease, previous myocardial infarction, cardiac arrhythmias, congestive heart failure, presence of pacemaker or defibrillator device, and previous coronary artery bypass grafting or percutaneous coronary intervention. Renal disease was defined as the presence of chronic kidney disease with or without the need for dialysis. Malignancy was defined as the presence of active or a history of previous malignancy. Smoking was defined as an active or remote history of smoking. Body mass index (BMI) was used to classify patients into normal weight (BMI 18.5-24.9 kg/m2), overweight (BMI $25-29.9 \mathrm{~kg} / \mathrm{m} 2$ ), obese (BMI $>=30 \mathrm{~kg} / \mathrm{m} 2$ ), and underweight (BMI $<18.5 \mathrm{~kg} / \mathrm{m} 2$ ) based on the classification by the World Health Organization. Obesity was further classified into Class I, Class II, and Class III based on WHO definitions.

\section{Outcome measures and statistical analysis}

The study was done according to STROBE guidelines for observational studies [5]. We computed mean, standard deviation, median with inter quartile range (IQR), frequency, and percentages as our descriptive variables. Differences in mean and percentage were assessed using the t-test and chi-squared test respectively. Differences in the median were calculated using the Mann-Whitney test.

Our main outcome was in-hospital mortality, hereby referred to as mortality. Length of hospital stay was recorded as a secondary outcome. Peak-CRP and peak-D-dimer levels were correlated with baseline patient characteristics, and analyzed as secondary outcomes.

To explore the risks associated with mortality, logistic regression analysis by univariate and age-adjusted models were performed for patient characteristics. Subsequently, to determine the independent association between these characteristics and mortality, multivariable logistic regression models were used to estimate odds ratios (ORs) adjusted for clinical covariates. Multivariable analysis was performed using the least absolute shrinkage and selection operator (Lasso) inference, a standard model building approach [6]. Lasso is most useful for sparse high-dimensional models such as ours. Cross-fit partialing-out, also known as double machine learning (DML), was used for estimation as this inference method is robust in identifying true variables among the specified potential control variable [7]. Stata version 16.0 was used for analysis (Stata Corp, Houston, TX). Age, sex, hypertension, diabetes, cardiac disease, and BMI class were always included as controls in this model by "forcing" Lasso unless one of these was the variable of interest. Lasso selected variables in the model from the following covariates [race, $\mathrm{NH}$ residency status, chronic obstructive pulmonary disease (COPD), renal disease, malignancy, and smoking status]. When one of these covariates was the variable of interest, it was not specified to Lasso for model selection. Two-tailed $\mathrm{p}<0.05$ was considered statistically significant.

Inflammatory over-reaction and endothelial damage leading to vasculitis and thrombosis have been described as prominent manifestations in critically ill COVID-19 patients. We collected information on markers of inflammation (peak-CRP) and thrombosis (peak-D-dimer). Data for peak-CRP were available in $164 / 348$ patients and for peak-D-dimer in 225/348 patients. We did not specify peak-CRP and peak-D-dimer as covariates in the Lasso inferential models as they are not the patients' baseline characteristics (demographics and comorbidities). A similar approach has been taken by other researchers while analyzing outcomes data in COVID-19 [8-9]. In addition, from a pathophysiologic standpoint, they are considered a 
consequence of the illness and perhaps a measure of disease severity [10]. The goal of our study was to identify high risk-comorbidities and demographic characteristics from all the baseline patient characteristics that portend a worse outcome (death). We were additionally interested in finding out the baseline characteristic that would affect the levels of peak-CRP and peak-D-dimer, essentially causing severe COVID-19. Indeed, peak-CRP and peak-D-dimer were considered an outcome in our study and identified as dependent variables. Age-adjusted and multivariable (Lasso inference) linear regression models were used to identify the effect of patient characteristics on peak-CRP and peak-D-dimer. Covariates used in the inferential model for peak-CRP and peak-D-dimer were the same as described above. Regression analysis for peak-CRP was done after excluding an outlier, serum value $1600 \mathrm{mg} / \mathrm{L}$ in a cohort with a range between 0 and $446 \mathrm{mg} / \mathrm{L}$. We then calculated age-adjusted and multivariable model-adjusted (Lasso inference) odds of mortality using peak-CRP and peak-D-dimer as the variables of interest. In addition to baseline characteristics described above, peak-CRP and peak-D-dimer levels were additionally used as covariates for this model, unless one of them was the variable of interest. Odds of mortality for peak-D-dimer were calculated after excluding the outliers (the lowest and the highest $10 \%$ values).

\section{Ethics}

The study was approved by the departmental research review committee with a waiver of informed consent due to its retrospective design (Approval number 20.5.06).

\section{Results}

Among the 348 patients, the median age was 71 years (IQR 59-82 years), 56.6\% were men, 33.6\% were white, and $36.2 \%$ presented from an NH (Table 1). Patients who died were significantly older than patients who survived (78 years vs 65 years, $\mathrm{p}<0.001$ ). The mortality rate among admissions from $\mathrm{NH}$ was $53.2 \%$, among those from the community was $33.3 \%$ and overall, $40.5 \%$. Not surprisingly, a significantly higher proportion of people who died were from $\mathrm{NH}(48 \%$ vs $29 \%$, $\mathrm{p}<0.001)$. The proportion of Caucasians among non-survivors was higher than that among survivors ( $40 \%$ vs $29 \%, \mathrm{p}=0.03$ ). This was because they were more likely to be from an $\mathrm{NH}$ ( $58 \%$ vs $25 \%$, p $<0.001)$ and were significantly older when compared to other races/ethnicities (76 years vs 67 years, $\mathrm{p}<0.001$ ). Patients who died were more likely to have hypertension and cardiac disease when compared to patients who survived. Peak-CRP and peak-D-dimer levels were significantly higher in non-survivors compared to survivors (Table 2 and Table 3). 


\section{Cureus}

\begin{tabular}{|c|c|c|c|c|}
\hline & Entire cohort $(n=348)$ & Survivors $(\mathrm{n}=207$ ) & Nonsurvivors ( $n=141$ ) & p value \\
\hline Age, years - median (IQR) & 71 (59-82) & $65(55-76)$ & 78 (69-87) & $<0.001$ \\
\hline Sex - Male, n (\%) & $197(56.6)$ & $120(57.9)$ & 77 (54.6) & 0.5 \\
\hline \multicolumn{5}{|l|}{ Race } \\
\hline Caucasian & $117(33.6)$ & $60(28.9)$ & $57(40.4)$ & 0.03 \\
\hline African American & $123(35.3)$ & $77(37.2)$ & 46 (32.6) & 0.4 \\
\hline Hispanic & $69(19.8)$ & $44(21.3)$ & $25(17.7)$ & 0.4 \\
\hline Asian & $8(2.3)$ & $6(2.9)$ & $2(1.4)$ & 0.4 \\
\hline Others & $31(8.9)$ & $20(9.7)$ & $11(7.8) 0$ & 0.6 \\
\hline NH status & $126(36.2)$ & $59(28.5)$ & $67(47.5)$ & $<0.001$ \\
\hline \multicolumn{5}{|l|}{ Comorbidities } \\
\hline Hypertension & $232(66.7)$ & $126(60.9)$ & 106 (75.2) & 0.01 \\
\hline Diabetes & 147 (42.2) & $81(39.1)$ & $66(46.8)$ & 0.2 \\
\hline Cardiac diseases & $113(32.5)$ & $57(27.5)$ & $56(39.7)$ & 0.02 \\
\hline COPD & $48(13.8)$ & 27 (13.04) & $21(14.9)$ & 0.6 \\
\hline Renal disease & 64 (18.4) & $39(18.8)$ & 25 (17.7) & 0.8 \\
\hline Malignancy & 67 (19.3) & 35 (16.9) & 32 (22.7) & 0.2 \\
\hline Smoking status & $62(17.8)$ & $37(17.9)$ & $25(17.7)$ & 0.9 \\
\hline \multicolumn{5}{|l|}{ Body habitus } \\
\hline Normal BMI & $104(29.9)$ & $64(30.9)$ & $40(28.4)$ & 0.6 \\
\hline Overweight & $123(35.3)$ & 70 (33.8) & $53(37.6)$ & 0.5 \\
\hline Obesity & 121 (34.8) & 73 (35.3) & 48 (34.04) & 0.8 \\
\hline Class -1 & 65 (18.7) & 39 (18.8) & 26 (18.4) & 0.9 \\
\hline Class - II & $33(9.5)$ & $20(9.7)$ & $13(9.2)$ & 0.9 \\
\hline Class - III & $23(6.6)$ & $14(6.8)$ & $9(6.4)$ & 0.9 \\
\hline LOS (Days + SD) & $8.2+7.3$ & $8.1+7.5$ & $8.4+7.1$ & 0.7 \\
\hline
\end{tabular}

\section{TABLE 1: Baseline characteristics of hospitalized COVID-19 patients.}

Data presented as number (percentage) except age and length of stay.

COVID-19, coronavirus disease 2019; BMI, body mass index; COPD, chronic obstructive pulmonary disease; IQR, inter quartile range; LOS, length of hospital stay; SD, standard deviation; $\mathrm{NH}$, nursing home.

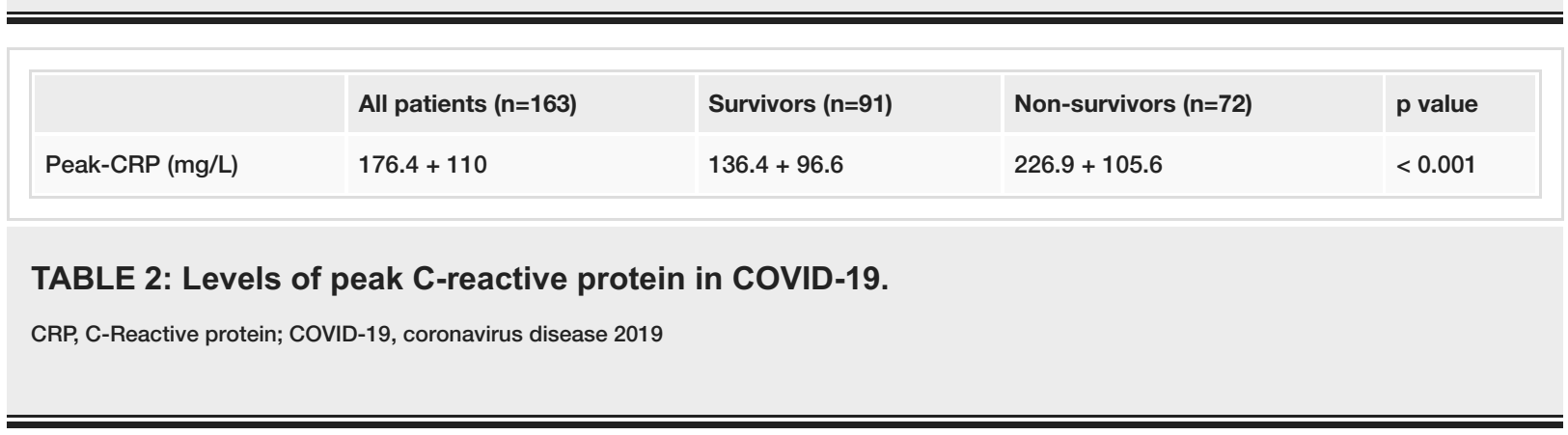




\section{Cureus}

Characteristic

Peak-D-dimer (mcg/mL), median (IQR)
All patients $(n=225)$

$1.5(0.5-4.7)$
Survivors $(n=123)$

$0.7(0.3-2.5)$
Non-survivors $(n=102)$

$2.9(1.1-14.2)$ p value

$<0.001$

TABLE 3: Levels of peak-D-dimer in COVID-19.

IQR, inter quartile range; COVID-19, coronavirus disease 2019

On univariate analysis, those who died of COVID-19 were more likely to be older, OR 1.06 (1.04-1.08) for being older by one year, $\mathrm{p}=<0.001$ (Table 4 ). Those who died of COVID-19 were also more likely to have hypertension and cardiac disease (Table 4). In the age-adjusted model, being overweight \{OR 1.9 (1.1-3.5) $\mathrm{p}=0.03\}$ and obese $\{\mathrm{OR} 2.5(1.3-4.6) \mathrm{p}=0.005\}$ correlated with mortality (Table 5). On multivariable logistic regression, patients who died had higher odds of being older $\{$ OR $1.06(1.04-1.1) \mathrm{p}<0.001\}$ and belonging to BMI classes - overweight \{OR $2(1.1-3.7) \mathrm{p}=0.03\}$ and obesity $\{$ OR $2.5(1.2-4.9) \mathrm{p}=0.01\}$.

\begin{tabular}{|c|c|c|}
\hline & OR (CI) & p value \\
\hline Age (years) & $1.06(1.04-1.08)$ & $<0.001$ \\
\hline Gender (Male vs Female) & $0.9(0.6-1.3)$ & 0.5 \\
\hline \multicolumn{3}{|l|}{ Race (reference Caucasian) } \\
\hline African American & $0.6(0.4-1 \cdot 1)$ & 0.1 \\
\hline Hispanic & $0.6(0.3-1 \cdot 1)$ & 0.1 \\
\hline Asian & $0.4(0.7-1 \cdot 8)$ & 0.2 \\
\hline Others & $0.6(0.3-1 \cdot 3)$ & 0.2 \\
\hline \multicolumn{3}{|l|}{ Comorbidities } \\
\hline Hypertension & $1.9(1.2-3.1)$ & 0.006 \\
\hline Diabetes & $1.4(0.9-2.1)$ & 0.2 \\
\hline Cardiac diseases & $1.7(1.1-2.7)$ & 0.02 \\
\hline COPD & $1.2(0.6-2.2)$ & 0.6 \\
\hline Renal disease & $0.9(0.5-1.6)$ & 0.8 \\
\hline Malignancy & $1.4(0.8-2.5)$ & 0.2 \\
\hline Smoking status & $0.9(0.6-1 \cdot 7)$ & 0.9 \\
\hline \multicolumn{3}{|c|}{ Body habitus (reference normal BMI) } \\
\hline Overweight & $1.2 \cdot(0.7-2.1)$ & 0.5 \\
\hline Obesity & $1.1(0.6-1.8)$ & 0.9 \\
\hline
\end{tabular}

TABLE 4: Univariate analysis of patient characteristics with mortality.

COPD, chronic obstructive pulmonary disease; $\mathrm{BMI}$, body mass index; OR, odds ratio; $\mathrm{Cl}$, confidence interval 


\section{Cureus}

\begin{tabular}{|c|c|c|c|c|}
\hline & Age-adjusted OR (Cl) & $p$ value & Multivariable OR (Cl) & $\mathrm{p}$ value \\
\hline Age & - & - & $1.06(1.04-1.1)$ & $<0.001$ \\
\hline Male sex (ref Female) & $1.0(0.8-1.9)$ & 0.4 & $1.2(0.8-2.02)$ & 0.4 \\
\hline \multicolumn{5}{|l|}{ Race (ref Caucasian) } \\
\hline African American & $0.9(0.5-1.5)$ & 0.6 & $0.8(0.5-1.5)$ & 0.5 \\
\hline Hispanic & $1.5(0.7-2.9)$ & 0.3 & $1.6(0.7-3.5)$ & 0.2 \\
\hline Asian & $0.5(0.1-2.8)$ & 0.4 & $0.6(0.1-3.1)$ & 0.5 \\
\hline Others & $0.8(0.3-1.9)$ & 0.6 & $0.8(0.3-1.8)$ & 0.5 \\
\hline \multicolumn{5}{|l|}{ Comorbidities } \\
\hline Hypertension & $1.3(0.8-2.2)$ & 0.3 & $1.2(0.7-2.1)$ & 0.6 \\
\hline Diabetes & $1.5(0.9-2.4)$ & 0.1 & $1.3(0.8-2.2)$ & 0.3 \\
\hline Cardiac diseases & $1.1(0.6-1.7)$ & 0.8 & $1.02(0.6-1.7)$ & 0.9 \\
\hline COPD & $0.8(0.4-1.6)$ & 0.6 & $0.8(0.4-1.7)$ & 0.6 \\
\hline Renal disease & $0.7(0.4-1.2)$ & 0.2 & $0.6(0.3-1.1)$ & 0.1 \\
\hline Malignancy & $1.1(0.6-1.9)$ & 0.8 & $1.1(0.6-1.9)$ & 0.9 \\
\hline Smoking status & $0.8(0.4-1.4)$ & 0.4 & $0.8(0.4-1.4)$ & 0.4 \\
\hline \multicolumn{5}{|l|}{ Body habitus } \\
\hline Normal BMI (ref) & - & - & - & - \\
\hline Overweight & $1.9(1.1-3.5)$ & 0.03 & $2(1.1-3.7)$ & 0.03 \\
\hline Obesity & $2.5(1.3-4.6)$ & 0.005 & $2.5(1.2-4.9)$ & 0.01 \\
\hline \multicolumn{5}{|l|}{ Sensitivity analysis for sex } \\
\hline \multicolumn{5}{|l|}{ Among Women } \\
\hline Overweight & $2.6(0.9-6.6)$ & 0.056 & $2.6(0.9-7.5)$ & 0.08 \\
\hline Obesity & $5.1(1.8-14.1)$ & 0.002 & $4.4(1.4-13.5)$ & 0.01 \\
\hline \multicolumn{5}{|l|}{ Among Men } \\
\hline Overweight & $1.5(0.7-3.2)$ & 0.3 & $1.5(0.6-3.6)$ & 0.3 \\
\hline Obesity & $1.5(0.6-3.5)$ & 0.4 & $1.4(0.5-3.6)$ & 0.5 \\
\hline
\end{tabular}

\section{TABLE 5: Risk factors for in-hospital mortality.}

$\mathrm{BMI}$, body mass index; COPD, chronic obstructive pulmonary disease; $\mathrm{Cl}$, confidence interval; OR, odds ratio; ref, reference.

Analysis by sex was done to assess the effect of sex on the association of BMI classes and mortality. Among women, those who died seemed to have higher odds of being overweight, although this did not reach predetermined statistical significance (Table 5). The presence of obesity in women was significantly correlated with mortality in both the age-adjusted and multivariable model \{OR 4.4 (1.4-13.5) p=0.01\}. Among men, no correlation was seen between in-hospital mortality and obesity (Table 5).

When further analyzed by the severity of obesity class, we found increasingly higher odds with progressively worsening obesity in a "dose-dependent" manner, although not always statistically significant (Table 6 and Figure 1). 


\section{Cureus}

\begin{tabular}{|c|c|c|c|c|}
\hline & Age-adjusted OR (Cl) & $p$ value & Multivariable OR (CI) & p value \\
\hline \multicolumn{5}{|l|}{ All patients } \\
\hline Normal weight $(n=104)$ & - & - & - & - \\
\hline Overweight ( $n=123)$ & $1.9(1.1-3.6)$ & 0.03 & $1.9(1.05-3.8)$ & 0.036 \\
\hline Obese class $-1(n=65)$ & $2.1(1.02-4.3)$ & 0.04 & $2.3(1.01-4.9)$ & 0.046 \\
\hline Obese class $-2(n=33)$ & $2.6(1.02-6.4)$ & 0.04 & $2.4(0.9-6.3)$ & 0.067 \\
\hline Obese class $-3(n=23)$ & $4.3(1.5-12.6)$ & 0.008 & $4.6(1.4-15.7)$ & 0.015 \\
\hline \multicolumn{5}{|l|}{ Women only } \\
\hline Normal (n=52) & - & - & - & - \\
\hline Overweight $(n=45)$ & $2.6(0.9-6.8)$ & 0.054 & $2.7(0.8-7.9)$ & 0.08 \\
\hline Obese class $-1(n=29)$ & $4.8(1.5-15.1)$ & 0.008 & $4.1(1.2-14.1)$ & 0.03 \\
\hline Obese class $-2(n=14)$ & $4.2(0.9-18.4)$ & 0.054 & $3.7(0.8-17.3)$ & 0.09 \\
\hline Obese class $-3(n=11)$ & $8.2(1.7-41)$ & 0.01 & $6.9(1.04-46.2)$ & 0.046 \\
\hline \multicolumn{5}{|l|}{ Men only } \\
\hline Normal (n=52) & - & - & - & - \\
\hline Overweight ( $n=78)$ & $1.5(0.7-3.3)$ & 0.3 & $1.7(0.7-4.3)$ & 0.3 \\
\hline Obese class $-1(n=36)$ & $1.2(0.5-3.1)$ & 0.7 & $1.2(0.4-3.7)$ & 0.7 \\
\hline Obese class $-2(n=19)$ & $1.8(0.6-6.1)$ & 0.3 & $1.9(0.5-7.2)$ & 0.3 \\
\hline Obese class - $3(n=12)$ & $2.9(0.6-12.9)$ & 0.2 & $3.3(0.7-16.9)$ & 0.2 \\
\hline
\end{tabular}

TABLE 6: Risk of in-hospital death with obesity stratified by severity classes.

n, number; OR, odds ratio; $\mathrm{Cl}$, confidence interval

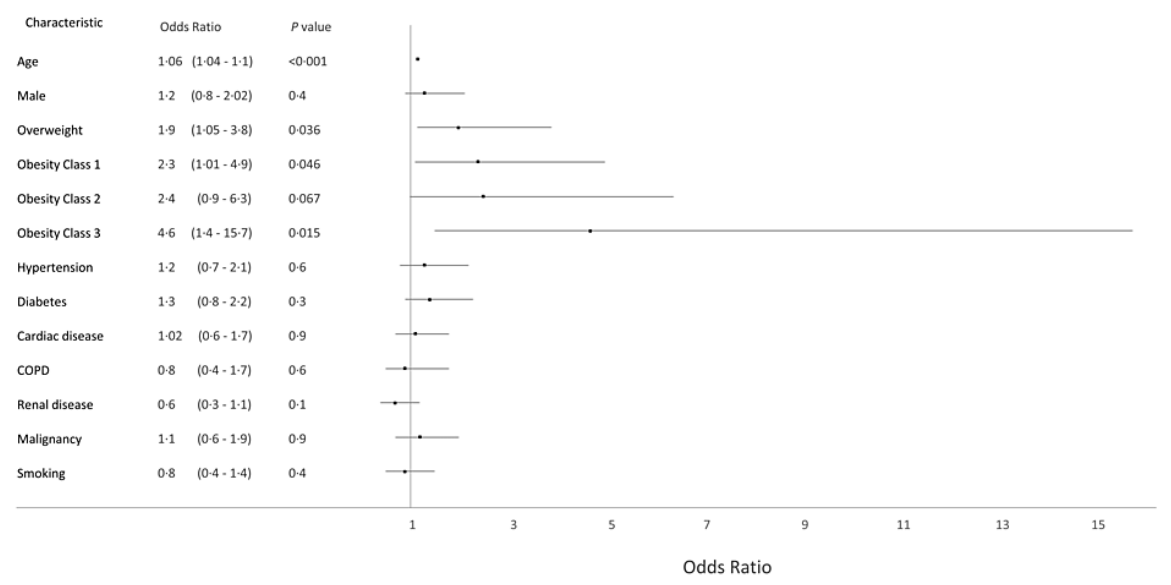

FIGURE 1: Forest plot showing adjusted odds of in-hospital mortality.

Again, this effect was not seen in men and was primarily because of an independent correlation between excess weight and mortality in women (Table 6, Figures 2-3). 


\section{Cureus}

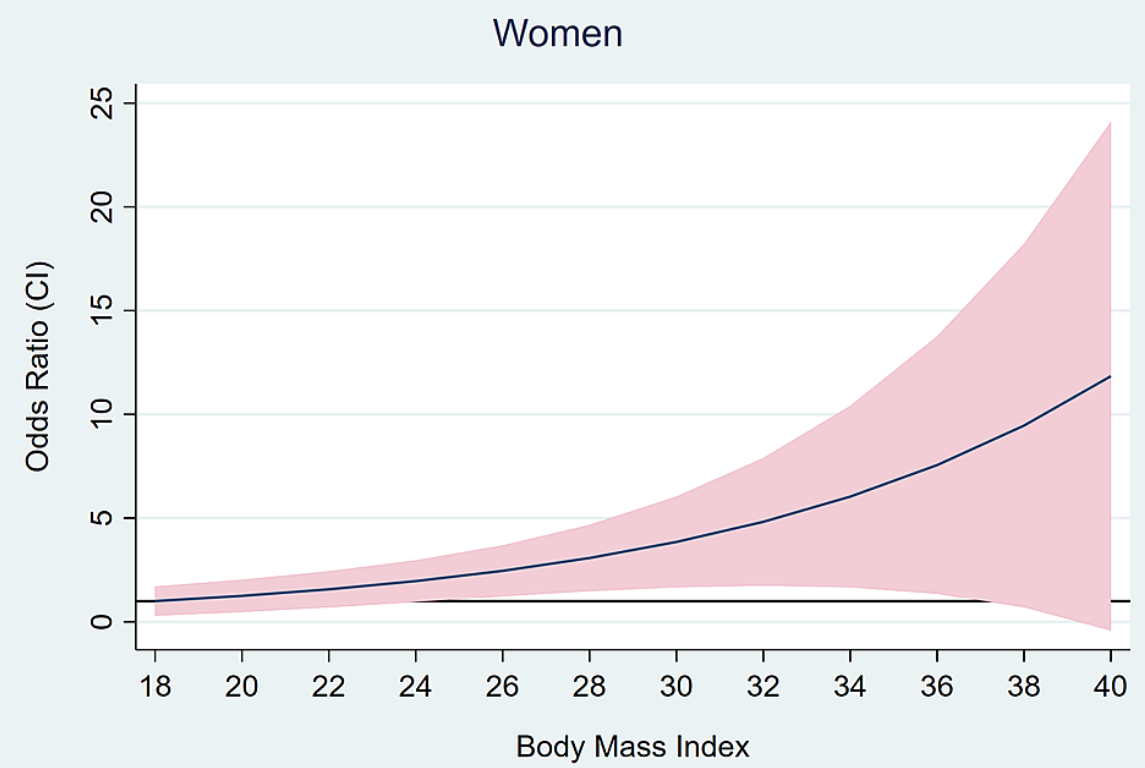

FIGURE 2: Age-adjusted odds of in-hospital mortality with increasing BMI in women.

BMI, body mass index

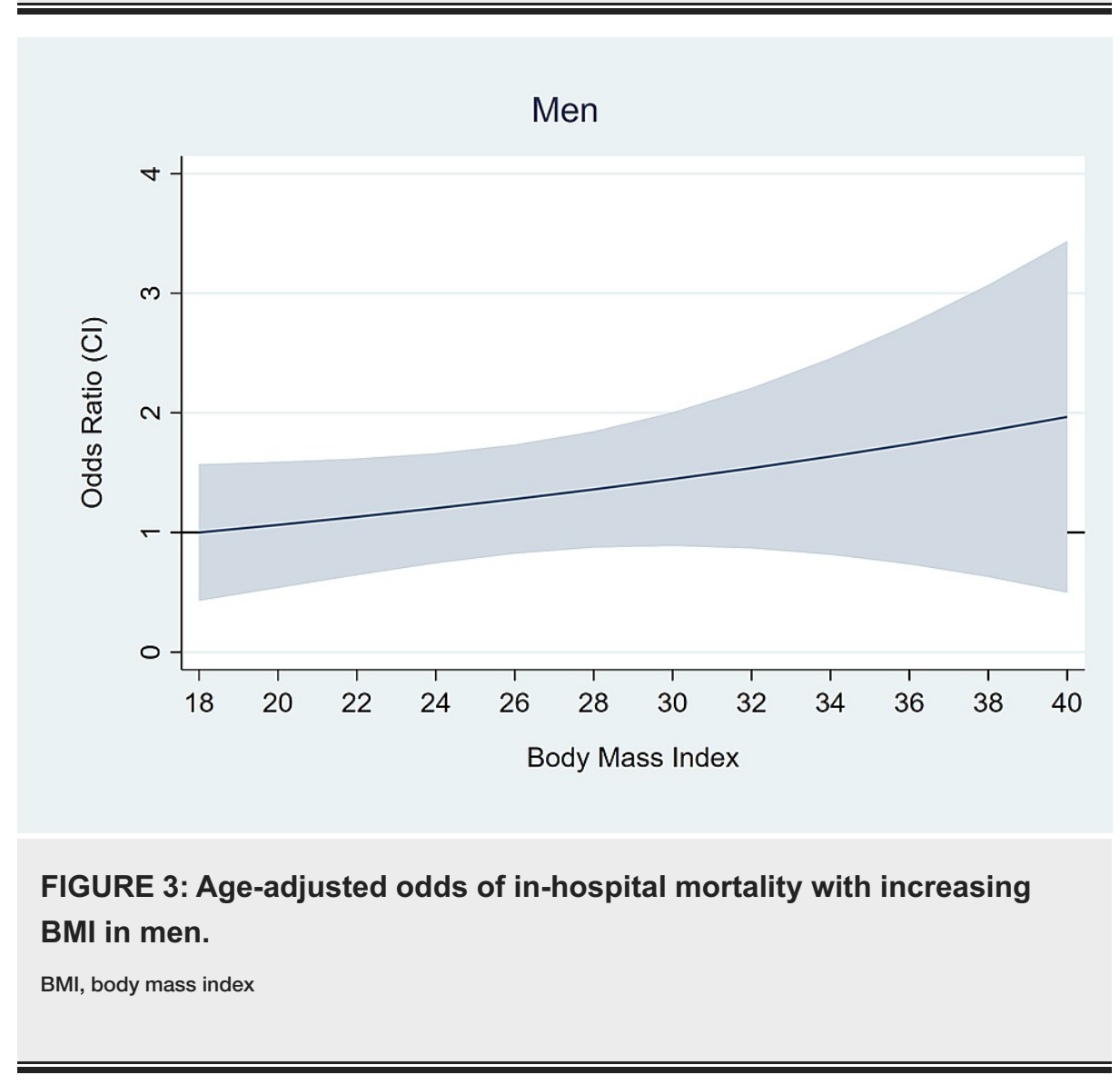

In the secondary outcome, obesity was the only patient characteristic that significantly correlated with peakCRP levels (Table 7). Race and hypertension correlated with peak-D-dimer levels while no such association with obesity was revealed (Table 8 ). Peak-CRP was associated with significantly higher odds of mortality, odds ranging between 1.5 and 1.6 for every $50 \mathrm{mg} / \mathrm{L}$ increase in peak-CRP levels $(p<0.001)$. Odds of mortality 


\section{Cureus}

were increased with increasing peak-D-dimer levels as well (Table 9).

\begin{tabular}{|c|c|c|c|c|}
\hline & Coefficient (age-adjusted model) & p value & Coefficient (multivariable model) & p value \\
\hline Age & - & - & $1.3(-0.1-2.7)$ & 0.06 \\
\hline Male gender & $16.8(-18.8-52.3)$ & 0.4 & $22.2(-12.1-56.6)$ & 0.2 \\
\hline \multicolumn{5}{|l|}{ Race (ref Caucasian) } \\
\hline African American & $0.4(-41.7-42.5)$ & 0.9 & $7.6(-31.9-47.1)$ & 0.7 \\
\hline Hispanic & $42.2(-7.2-91.6)$ & 0.09 & $48.4(-3.5-100.4)$ & 0.07 \\
\hline Asian & $-77.6(-205-49.9)$ & 0.2 & $-72.5(-172.6-27.6)$ & 0.2 \\
\hline Others & $-41.3(-104.1-21.6)$ & 0.2 & $-25.5(-79.8-28.9)$ & 0.4 \\
\hline \multicolumn{5}{|l|}{ Comorbidities } \\
\hline Hypertension & $-3.8(-42.3-34.8)$ & 0.9 & $3.8(-35.9-43.5)$ & 0.9 \\
\hline Diabetes & $-32.3(-66.6-1.9)$ & 0.07 & $-27.7(-63.4-8)$ & 0.1 \\
\hline Cardiac disease & $-44.2(-81.6--6.8)$ & 0.02 & $-37.4(-75-0.2)$ & 0.05 \\
\hline COPD & $-17.3(-70.1-35.4)$ & 0.5 & $-9.8(-61-41.5)$ & 0.7 \\
\hline Renal disease & $-37.8(-83.3-7.6)$ & 0.1 & $-20(-66.7-26.7)$ & 0.4 \\
\hline Malignancy & $-12.6(-57.8-32.7)$ & 0.6 & $-9.8(-53.7-34.1)$ & 0.7 \\
\hline Smoking status & $3.3(-44.6-51.1)$ & 0.9 & $13.1(-34.5-60.7)$ & 0.6 \\
\hline \multicolumn{5}{|c|}{ Body habitus (ref normal BMI) } \\
\hline Overweight & $19.2(-24.3-62.6)$ & 0.4 & $18.9(-20.8-58.6)$ & 0.4 \\
\hline Obesity & $45.4(0.4-90.5)$ & 0.048 & $45.1(5.9-84.3)$ & 0.02 \\
\hline
\end{tabular}

TABLE 7: Predictors of peak-CRP levels (measured in milligrams per liter) by linear regression analysis.

COPD, chronic obstructive pulmonary disease; BMI, body mass index; ref, reference; CRP, C-reactive protein 


\section{Cureus}

\begin{tabular}{|c|c|c|c|c|}
\hline & Coefficient (age-adjusted model) & p value & Coefficient (multivariable model) & p value \\
\hline Age & - & - & $0.1(0.005-0.2)$ & 0.04 \\
\hline Male gender & $1.3(-12.4-3.8)$ & 0.3 & $1.3(-1.1-3.8)$ & 0.3 \\
\hline \multicolumn{5}{|l|}{ Race (ref Caucasian) } \\
\hline African American & $5.4(2.5-8.3)$ & $<0.001$ & $5.3(2.3-8.3)$ & 0.001 \\
\hline Hispanic & $3.4(-0.0004-6.8)$ & 0.05 & $3.9(0.8-6.9)$ & 0.01 \\
\hline Asian & $0.6(-7.6-8.8)$ & 0.9 & $0.8(-4.2-5.9)$ & 0.8 \\
\hline Others & $-0.7(-5.2-3.8)$ & 0.8 & $-0.3(-2.8-2.2)$ & 0.8 \\
\hline \multicolumn{5}{|l|}{ Comorbialties } \\
\hline Hypertension & $2.9(0.2-5.7)$ & 0.03 & $3.5(0.9-5.9)$ & 0.006 \\
\hline Diabetes & $-0.2(-2.7-2.3)$ & 0.9 & $-1.1(-3.8-1.6)$ & 0.4 \\
\hline Cardiac disease & $-1.4(-4.2-1.3)$ & 0.3 & $-0.7(-3.7-1.7)$ & 0.5 \\
\hline COPD & $-0.8(-4.5-2.9)$ & 0.7 & $-0.3(-3.9-3.2)$ & 0.9 \\
\hline Renal disease & $-2.5(-5.7-0.7)$ & 0.1 & $-2.8(-5.7-0.0009)$ & 0.05 \\
\hline Malignancy & $-1.7(-4.9-1.5)$ & 0.3 & $-1.3(-4.3-1.8)$ & 0.4 \\
\hline Smoking status & $-1.9(-5.2-1.3)$ & 0.2 & $-2.1(-5.1-0.8)$ & 0.2 \\
\hline \multicolumn{5}{|c|}{ Body habitus (ref normal BMI) } \\
\hline Overweight & $1.5(-1.6-4.6)$ & 0.4 & $1.6(-1.5-4.7)$ & 0.3 \\
\hline Obesity & $2.3(-1.01-5.6)$ & 0.2 & $1.8(-1.3-4.9)$ & 0.3 \\
\hline
\end{tabular}

TABLE 8: Predictors of peak-D-dimer levels (measured in $\mathrm{mcg} / \mathrm{mL}$ ) by linear regression analysis.

COPD, chronic obstructive pulmonary disease; BMI, body mass index; ref, reference

\begin{tabular}{|c|c|c|c|c|}
\hline Characteristic & Age-adjusted OR (CI) & p value & Multivariable model OR (Cl) & $p$ value \\
\hline CRP (every 50 mg/L increase) & $1.6(1.3-1.9)$ & $<0.001$ & $1.5(1.3-1.8)$ & $<0.001$ \\
\hline D-dimer (every $0.5 \mathrm{mcg} / \mathrm{mL}$ increase) & $1.06(1.03-1.1)$ & $<0.001$ & $1.05(1.02-1.1)$ & $<0.001$ \\
\hline CRP, C-Reactive protein; OR, odds ratio; C & nce interval & & & \\
\hline
\end{tabular}

\section{Discussion}

In this study, we present data identifying patient characteristics that portend a worse prognosis for patients hospitalized with COVID-19. Our patient cohort was diverse; with whites, blacks, and other races comprising a third of the patients each. We found significantly higher odds of mortality with increasing age. Every one-year increment in age increased the odds of death by 1.06. This is not surprising, as by now advancing age is an established risk factor for poor outcomes in COVID-19 [9, 11]. Besides age, the only other patient characteristic that impacted mortality was the presence of excess weight. Patients who died had more than two-fold odds of being overweight and obese compared to the people who survived. This effect was seen in both age-adjusted and a robust multivariable model built using a standardized approach (Lasso inference). The correlation between excess weight and mortality manifested as if it were a "doseeffect” response, i.e. in those who died progressively higher odds of belonging to a higher BMI class were observed, compared to those who survived (Figure 1). Interestingly this correlation between mortality and excess weight was seen only in women, with as much as four to five times higher odds of being afflicted by 
excess weight in patients who died compared to those who survived (Figure 2). The excess weight did not seem to have an effect on mortality among men, as increasing age was the only identifiable risk among men (Figure 3). It is noteworthy that in those who died, the odds for Obesity Class II failed to meet the prerequisite statistical significance of $\mathrm{p}<0.05$. We believe it is likely a result of a small sample size leading to a lack of power in detecting a significance. This is also reflected in the wide confidence intervals seen in the odds. Similarly, we believe other risk factors such as diabetes and hypertension did not meet statistical significance likely from a lack of power due to the small sample size.

In previous influenza and novel coronavirus epidemics, severe acute respiratory syndrome (SARS-CoV-1) and the Middle East respiratory syndrome (MERS), in addition to virus-induced cytopathic effects an excessive and dysregulated host immune response played a crucial role in the pathology and mortality [1213]. This exaggerated response with supraphysiologic levels of cytokines is referred to as cytokine release syndrome or "cytokine-storm" [14-15]. Cytokine-storm plays a crucial role in COVID-19 severity and outcome [4, 14-15]. Peripheral blood levels of interleukin-6 and C-Reactive protein (CRP) correlate well with the onset and presence of cytokine storm [14]. CRP in contrast to interleukin-6 is a widely available test in most hospitals. CRP was recently shown to correlate with the extent of lung pathology and disease severity in COVID-19 patients [10].

Obesity has been increasingly identified as a risk factor for worse outcomes in COVID-19 [8-9]. Earlier data from China did not reveal this effect probably because the prevalence of obesity in China is significantly lower (6\%) when compared to the United States (42\%) and Europe (>20\%) [9]. Data from NYC suggested that obesity might be a risk factor for ICU admission in younger patients (age <60 years) with COVID-19 [16]. Increased independent odds of mortality with obesity have been described in COVID-19 patients [9]. However, a handful of studies before us have provided analysis on odds of mortality by obesity class [17]. This association of a noncommunicable illness with outcomes of viral epidemics is not surprising or new. Obesity has been previously recognized as a risk factor for severe illness, hospitalization, and death in the 2009 influenza pandemic (H1N1) as well as during the MERS epidemic [18-19].

Our finding begs the question, what are the pathophysiologic mechanisms behind this association? Generally, individuals affected by obesity demonstrate a restrictive pattern on pulmonary function testing, have impairment of ventilation due to impedance to diaphragmatic excursions, possess reduced lung volumes, and are more likely immunosuppressed from often associated diabetes, increasing their risk of pneumonia [20].

Adipose tissue should not be considered a mere storage site of excess energy, as it plays a more complex physiologic role through endocrine and immunologic responses. Adipose tissue consists of preadipocytes, adipocytes, infiltrated B-cells, T-cells, macrophages, and neutrophils [21-22]. Adipose tissue is essentially an immunologically active organ that displays hallmarks of both innate and adaptive immune responses [21]. Obesity increases the immune cells in adipose tissue and skews their phenotype from anti-inflammatory towards more pro-inflammatory subtypes [21-22]. Thereby, obesity increases inflammation, makes the immune system more vulnerable to infections, and less responsive to vaccinations, antivirals, and antimicrobials [22]. As an example, in influenza, individuals afflicted by obesity have been shown to have high viral titers in the lung, worsened lung pathology, and increased mortality despite vaccination. By the above mechanisms, obesity causes a persistent elevation in inflammatory markers. Elevated interleukin-6, CRP, adipokines, cytokines, and interferons in obesity characterize a chronic low-grade inflammation [23]. Thus, obesity is interlinked with infections and inflammation in a manner that is far more complex than previously thought. In addition, adipocytes express angiotensin-converting enzyme 2 (ACE2) which is reportedly the mode of cellular entry for SARS-CoV-2 [24]. Therefore, a direct proinflammatory role of SARS-CoV-2 in intrapulmonary adipocytes should be considered as a mechanism of injury.

Thereby, increased adipose tissue in obese individuals could lead to an exaggerated inflammatory response and cytokine-storm in COVID-19. Indeed, obesity correlated with peak-CRP levels in our study while none of the other patient characteristics did. Unsurprisingly, peak-CRP was associated with mortality in our study, thereby supporting the pathologic role of a hyperactive inflammatory response. On the other hand, we found no association between obesity and peak-D-dimer levels. The effect of peak-D-dimer levels on mortality was significant but less pronounced than that of peak-CRP levels.

The patient's sex did not impact the outcome of mortality in our study. In general, men experience a greater severity and prevalence of bacterial, viral, fungal, and parasitic infections than women. Women also display a more robust response to antigenic challenges such as infection and vaccination [25]. This stronger immune response in women could, however, more frequently evolve into cytokine-storm contributing to worse outcomes. During viral epidemics, the sex difference in outcomes thus depends on the balance between the beneficial anti-viral effect and the harm from an exaggerated inflammatory response. Historically, influenza epidemics have varied in their risk to the sexes, some were worse for men (1918) and others for women (2004) [26]. In previous coronavirus outbreaks (SARS-CoV-1 and MERS), men had higher mortality rates than women [13, 27-28]. Likewise, COVID-19 hospitalizations have mostly comprised men, and thus far into the pandemic, men have been identified to be at greater risk [8-9]. As mentioned earlier, a difference in mortality with sex was not seen in our study. Our patient cohort was fairly old (3/4th of patients aged >=60 years) and it is possible that the effect of advancing age on mortality overpowered that of sex. Notably, a 
majority of studies have reported early mortality data while a significant proportion of patients were still hospitalized. Thus, mortality rates and outcomes may change when definite outcomes are analyzed by including those patients who were still hospitalized at the time of reporting. Meanwhile, some researchers have shared findings similar to ours, with no difference in mortality rates between the two sexes [11].

Prior to our study, most researchers did not perform a sensitivity analysis looking for the effect of sex on the association between obesity and mortality in COVID-19 [8]. To the best of our knowledge, only a handful of studies have analyzed and reported sex-disparity in the effect of obesity on outcomes in COVID-19 patients $[23,29]$. Interestingly, the studies found conflicting results. Researchers from California found an increased risk of obesity in men but not in women [29]. In contrast in a large cohort of patients from the UK, a higher BMI was associated with a stronger risk of COVID-19 mortality in women than in men; the womento-men ratio of hazard ratios was 1.20 (1.00-1.43) [23]. Similar to the study from the UK, we found a sexbased difference in the effect of obesity on mortality with a higher BMI increasing the odds of death for women but not for men. One of the reasons for this increased risk in women might be because obesity as defined by the BMI, does not correlate well with the adipose tissue mass, as BMI does not take body fat percentage into account. Women with a higher fat percentage have a higher mass of adipose tissue compared to men for the same body weight and BMI class. A greater amount of adipose tissue could mean a greater inflammatory response in the setting of COVID-19 illness leading to poor outcomes. However, our test for this hypothesis failed to show any difference in the peak-CRP levels between men and women, indicating that other forces could be at play. Previously, researchers identified a set of genes regulated by testosterone and participating in lipid biosynthesis, whose expression was negatively associated with antibody responses to influenza vaccine in men [25]. Higher testosterone levels corresponded with lower antibody response to the vaccine. This demonstrable association between sex hormones, genes involved in lipid metabolism, and altered immune response to viral antigens, gives us some insight into the rather complex associations between sex, lipid metabolism, and immunity. It is unclear if the genes involved in lipid biosynthesis had any correlation to the BMI. Further studies are needed to test these hypotheses. Our study adds to the literature supporting a higher risk with increasing BMI in women with COVID-19 as compared to men with COVID-19.

\section{Relevance of findings}

Clarifying the impact of obesity on COVID-19 severity and outcomes is of major public health importance. The last known prevalence of obesity in the United States was $42.4 \%$ in $2017-2018$ and its impact was seen in all age groups. Similar to the United States, other western countries have a high prevalence of obesity as well [9]. It would be a mistake to think that developing nations are relatively protected from the impact of obesity, and thus from a potentially increased death due to COVID-19. The largest study to date demonstrated that about two-thirds of the obese population in the world was living in developing countries [30]. If our findings are generalizable, the growing spread of the illness in developing nations such as Brazil and India is of monumental concern.

Our study adds to the growing literature supporting an almost dose-effect response between excess weight and mortality $[8,17]$. To the best of our knowledge, we are one of a handful of studies to report a sex disparity in this effect of excess weight on mortality [23, 29]. Due to conflicting results in the previous studies, further analyses and research is urgently needed to identify the population at risk. Given our findings and those from Lighter et al. and Peters et al., obesity could increase the risk to patient groups previously considered at low-risk, i.e. women and younger individuals [16, 23]. Unquestionably our findings are of great public health importance and need to be disseminated, corroborated, and elucidated.

In the interim, we recommend that people with obesity, especially women affected by severe obesity, should take extra precautions to avoid COVID-19 contamination by strictly enforcing social distancing and other prevention measures. Once diagnosed, patients with severe obesity should be monitored more closely. As the primary mechanism for death due to obesity is likely the immune over-reaction, these patients may benefit to a greater extent from immunomodulatory treatments aimed at the complement system or interleukin-6, which are being studied in clinical trials.

\section{Limitations}

The limitations of this study are that the study population was from a single hospital and included older patients, often from NHs, with multiple comorbidities and thus findings may not be broadly applicable to patients of different age groups. On the other hand, there are several communities spread across the United States and the world, with population characteristics similar to ours, and our experience should be directly applicable to them. Underweight patients were not well represented in our patient cohort; thus, we could not investigate outcomes in that subgroup of patients. Additionally, this is a retrospective case series and as such can only report on associations between the variables under study and cannot draw conclusions related to cause and effect. As the pandemic spread in our community and the rest of the world, and new information concerning the pathogenetic importance of cytokine storm and thrombosis became widely available, our practice patterns changed. Notably, a protocol for steroid use for cytokine-storm syndrome and anticoagulation for elevated D-dimers was adopted at our hospital and evolved as more information became available with the evolving pandemic. This most certainly was true for all the previous reports of 
COVID-19 as well, and in our estimation was not unique to our study.

\section{Conclusions}

In a diverse patient cohort, advancing age and obesity were independent predictors of mortality among hospitalized COVID-19 patients. While advancing age affected both sexes similarly, obesity impacted mortality among women but not among men. The association of obesity with mortality was more powerful than that of race, and all other comorbidities including hypertension, diabetes, and cardiac diseases. Out of all the patient characteristics, only obesity independently affected the blood levels of peak-CRP in COVID19 patients. This strengthens the hypothesis that a dysregulated and hyperactive immune response could be the central mechanism for increased risk among obese individuals. Based on our findings, immunomodulators might have higher efficacy in women affected by obesity. Further studies analyzing definite outcomes in COVID-19 are much needed. Confirmation of this increased risk with further data will help guide recommendations for the prevention, hospitalization, and management of these at-risk individuals. Our findings are of great public health importance and should be shared, corroborated, and elucidated.

\section{Additional Information \\ Disclosures}

Human subjects: Consent was obtained or waived by all participants in this study. Montefiore New Rochelle Medicine Research Review Committee issued approval 20.5.06. The study was approved by the departmental research review committee with a waiver of informed consent due to its retrospective design. Animal subjects: All authors have confirmed that this study did not involve animal subjects or tissue. Conflicts of interest: In compliance with the ICMJE uniform disclosure form, all authors declare the following: Payment/services info: All authors have declared that no financial support was received from any organization for the submitted work. Financial relationships: All authors have declared that they have no financial relationships at present or within the previous three years with any organizations that might have an interest in the submitted work. Other relationships: All authors have declared that there are no other relationships or activities that could appear to have influenced the submitted work.

\section{Acknowledgements}

Authors acknowledge Dr Maniraj Neupane, University of Pittsburgh Medical Center, for his guidance in the study design and interpretation of results. We acknowledge all health-care workers involved in the diagnosis and treatment of patients at our hospital.

\section{References}

1. COVID-19 dashboard by the Center for Systems Science and Engineering (CSSE) at Johns Hopkins . (2020). Accessed: April 20, 2021: https://coronavirus.jhu.edu/map.html.

2. In an early US coronavirus hot spot, business slowly reopens . (2020). Accessed: April 20, 2021: https://apnews.com/article/virus-outbreak-us-news-new-york-city-new-york-ny-state-wire9837010e732fc509c96071bcf7de8202.

3. Huang C, Wang Y, Li X, et al.: Clinical features of patients infected with 2019 novel coronavirus in Wuhan, China. Lancet. 2020, 395:497-506. 10.1016/S0140-6736(20)30183-5

4. Chiappetta S, Sharma AM, Bottino V, Stier C: COVID-19 and the role of chronic inflammation in patients with obesity. Int J Obes (Lond). 2020, 44:1790-1792. 10.1038/s41366-020-0597-4

5. von Elm E, Altman DG, Egger M, Pocock SJ, Gøtzsche PC, Vandenbroucke JP: The Strengthening of the Reporting of Observational Studies in Epidemiology (STROBE) statement: guidelines for reporting observational studies. J Clin Epidemiol. 2008, 61:344-349. 10.1016/j.jclinepi.2007.11.008

6. Tibshirani R: Regression shrinkage and selection via the Lasso. J R Stat Soc Ser B (Methodol). 1996, 58:267288. 10.1111/j.2517-6161.1996.tb02080.x

7. Chernozhukov V, Chetverikov D, Demirer M, et al.: Double/debiased machine learning for treatment and structural parameters. Econom J. 2018, 21:C1-C68. 10.1111/ectj.12097

8. Williamson EJ, Walker AJ, Bhaskaran K, et al.: Factors associated with COVID-19-related death using OpenSAFELY. Nature. 2020, 584:430-436. 10.1038/s41586-020-2521-4

9. Docherty AB, Harrison EM, Green CA, et al.: Features of 20133 UK patients in hospital with covid-19 using the ISARIC WHO Clinical Characterisation Protocol: prospective observational cohort study. BMJ. 2020, 369:m1985. 10.1136/bmj.m1985

10. Wang G, Wu C, Zhang Q, et al.: C-Reactive protein level may predict the risk of COVID-19 aggravation . Open Forum Infect Dis. 2020, 7:ofaa153. 10.1093/ofid/ofaa153

11. Zhou F, Yu T, Du R, et al.: Clinical course and risk factors for mortality of adult inpatients with COVID-19 in Wuhan, China: a retrospective cohort study. Lancet. 2020, 395:1054-1062. 10.1016/S01406736(20)30566-3

12. Liu Q, Zhou YH, Yang ZQ: The cytokine storm of severe influenza and development of immunomodulatory therapy. Cell Mol Immunol. 2016, 13:3-10. 10.1038/cmi.2015.74

13. Channappanavar R, Fett C, Mack M, Ten Eyck PP, Meyerholz DK, Perlman S: Sex-based differences in susceptibility to severe acute respiratory syndrome coronavirus infection. J Immunol. 2017, 198:4046-4053. 10.4049/jimmunol.1601896

14. Zhu Z, Cai T, Fan L, Lou K, Hua X, Huang Z, Gao G: Clinical value of immune-inflammatory parameters to 
assess the severity of coronavirus disease 2019. Int J Infect Dis. 2020, 95:332-339. 10.1016/j.ijid.2020.04.041

15. Mehta P, McAuley DF, Brown M, Sanchez E, Tattersall RS, Manson JJ: COVID-19: consider cytokine storm syndromes and immunosuppression. Lancet. 2020, 395:1033-1034. 10.1016/S0140-6736(20)30628-0

16. Lighter J, Phillips M, Hochman S, Sterling S, Johnson D, Francois F, Stachel A: Obesity in patients younger than 60 years is a risk factor for COVID-19 hospital admission. Clin Infect Dis. 2020, 71:896-897. 10.1093/cid/ciaa415

17. Kompaniyets L, Goodman AB, Belay B, et al.: Body mass index and risk for COVID-19-related hospitalization, intensive care unit admission, invasive mechanical ventilation, and death - United States, March-December 2020. Morb Mortal Wkly Rep. 2021, 70:355-361. 10.15585/mmwr.mm7010e4

18. Louie JK, Acosta M, Samuel MC, Schechter R, Vugia DJ, Harriman K, Matyas BT: A novel risk factor for a novel virus: obesity and 2009 pandemic influenza A (H1N1). Clin Infect Dis. 2011, 52:301-312. 10.1093/cid/ciq152

19. Al-Tawfiq JA, Hinedi K, Ghandour J, Khairalla H, Musleh S, Ujayli A, Memish ZA: Middle East respiratory syndrome coronavirus: a case-control study of hospitalized patients. Clin Infect Dis. 2014, 59:160-165. 10.1093/cid/ciu226

20. Afshin A, Forouzanfar MH, Reitsma MB, et al.: Health effects of overweight and obesity in 195 countries over 25 years. N Engl J Med. 2017, 377:13-27. 10.1056/NEJMoa1614362

21. Grant RW, Dixit VD: Adipose tissue as an immunological organ. Obesity (Silver Spring). 2015, 23:512-518. 10.1002/oby.21003

22. Dhurandhar NV, Bailey D, Thomas D: Interaction of obesity and infections. Obes Rev. 2015, 16:1017-1029. 10.1111/obr.12320

23. Peters SAE, MacMahon S, Woodward M: Obesity as a risk factor for COVID-19 mortality in women and men in the UK biobank: comparisons with influenza/pneumonia and coronary heart disease. Diabetes Obes Metab. 2021, 23:258-262. 10.1111/dom.14199

24. Patel VB, Basu R, Oudit GY: ACE2/Ang 1-7 axis: a critical regulator of epicardial adipose tissue inflammation and cardiac dysfunction in obesity. Adipocyte. 2016, 5:306-311. 10.1080/21623945.2015.1131881

25. Furman D, Hejblum BP, Simon N, et al.: Systems analysis of sex differences reveals an immunosuppressive role for testosterone in the response to influenza vaccination. Proc Natl Acad Sci USA. 2014, 111:869-874. 10.1073/pnas.1321060111

26. WHO: sex, gender and influenza in achieving gender equality and health equity . (2010). Accessed: April 20, 2021: https://apps.who.int/iris/bitstream/handle/10665/44401/9789241500111_eng.pdf.

27. Karlberg J, Chong DS, Lai WY: Do men have a higher case fatality rate of severe acute respiratory syndrome than women do?. Am J Epidemiol. 2004, 159:229-231. 10.1093/aje/kwh056

28. Alghamdi IG, Hussain II, Almalki SS, Alghamdi MS, Alghamdi MM, El-Sheemy MA: The pattern of Middle East respiratory syndrome coronavirus in Saudi Arabia: a descriptive epidemiological analysis of data from the Saudi Ministry of Health. Int J Gen Med. 2014, 7:417-423. 10.2147/IJGM.S67061

29. Tartof SY, Qian L, Hong V, et al.: Obesity and mortality among patients diagnosed with COVID-19: results from an integrated health care organization. Ann Intern Med. 2020, 173:773-781. 10.7326/M20-3742

30. Ng M, Fleming T, Robinson M, et al.: Global, regional, and national prevalence of overweight and obesity in children and adults during 1980-2013: a systematic analysis for the Global Burden of Disease Study 2013. Lancet. 2014, 384:766-781. 10.1016/S0140-6736(14)60460-8 